Research Article

\title{
Genome-wide identification and characterization of R2R3-MYB genes in Medicago truncatula
}

\author{
Wei $\mathrm{Li}^{1 *}$, Ying Liu ${ }^{1 *}$, Jinyue Zhao ${ }^{1}$, Xin Zhen ${ }^{1}$, Changhong Guo ${ }^{1}$ and Yongjun Shu ${ }^{1}$ iD \\ ${ }^{1}$ College of Life Science and Technology, Harbin Normal University, Harbin Heilongjiang, China.
}

\begin{abstract}
MYB is a large family of plant transcription factors. Its function has been identified in several plants, while there are few reports in Medicago truncatula. In this study, we used RNA-seq data to analyze and identify R2R3-MYB genes in the genome of Medicago truncatula. Phylogenetic analysis classified 150 MtMYB genes into 21 subfamilies with homologs. Out of the 150 MtMYB genes, 139 were distributed among 8 chromosomes, with tandem duplications (TD) and segment duplications (SD). Microarray data were used for functional analysis of the MtMYB genes during growth and developmental processes providing evidence for a role in tissues differentiation, seed development processes, and especially the nodulation process. Furthermore, we investigated the expression of MtMYB genes in response to abiotic stresses using RNA-seq data, which confirmed the critical roles in signal transduction and regulation processes under abiotic stress. We used quantitative real-time PCR (qRT-PCR) to validate expression profiles. The expression pattern of $M$. truncatula MYB genes under different abiotic stress conditions suggest that some may play a major role in cross-talk among different signal transduction pathways in response to abiotic stresses. Our study will serve as a foundation for future research into the molecular function of $M$. truncatula R2R3-MYB genes.
\end{abstract}

Keywords: Medicago truncatula, MYB, abiotic stress, qRT-PCR.

Received: August 16, 2018; Accepted: December 28, 2018.

\section{Introduction}

Myeloblastosis (MYB) genes are one of the largest transcription factors families in the plant kingdom (Romero et al., 1998; Rosinski and Atchley, 1998). MYB typically contain an MYB-binding domain at the N-terminus, composed of 1-4 imperfect repeats, with approximately 51-52 amino acid residues of incomplete conserved peptides encoding three $\alpha$-helices (Kanei-Ishii et al., 1990; Lipsick, 1996; Stracke et al., 2001). These three $\alpha$-helices form a helix-turn-helix (HTH) structure and fold with three relatively conserved tryptophan residues, separated by 18-19 amino acid residues of regular arrangement, and further participate in the formation of hydrophobic interactions (Ogata et al., 1995). The structure of the MYB domain reveals that the HTH interacts with the major groove of DNA (Ogata et al., 1994). MYB-containing genes have a diverse number of MYB proteins containing incomplete repeats and are divided into four categories, 1R-MYB, R2R3MYB, R1R2R3-MYB, and 4R-MYB (Jin and Martin, 1999).

Send correspondence to Yongjun Shu. College of Life Science and Technology, Harbin Normal University, No. 1 of Shida Road, 150025, Harbin, Heilongjiang, China. E-mail: syjun2003@126.com.

* These authors contributed equally to this study.
In the MYB gene family, R2R3-MYB is the largest category in plants and yeast (Martin and Pazares, 1997; Jin and Martin, 1999). To date, 126 R2R3-MYB genes have been identified in Arabidopsis (Stracke et al., 2001), 157 in maize (Du et al., 2012a), 244 in soybean (Du et al., 2012b), 205 in Gossypium raimondii (He et al., 2016), and 166 in cassava (Liao et al., 2016). R2R3-MYB transcription factors are involved with abiotic stress response, reactive oxygen species signaling pathways, secondary metabolism, and hormone signaling pathways. AtMYB41 from Arabidopsis is induced in response to high salinity, drought, cold, and abscisic acid (Lippold et al., 2009). AtMYB102 are rapidly induced by osmotic stress and abscisic acid (ABA) treatment (Denekamp and Smeekens, 2003). OsMYB55 is induced in rice by high temperature and over-expression of OsMYB55 resulted in improved plant growth under high temperature (Elkereamy et al., 2012). Meanwhile, OsMYB91 plays a role in plant growth regulation and salt stress tolerance in rice (Zhu et al., 2015). TaMYB4 of wheat is induced by salicylic acid, ethylene, abscisic acid, and methyl jasmonate, demonstrating a role of TaMYB4 in response to biotic stress (Al-Attala et al., 2014). GbMYB5 from Gossypium barbadense is positively involved in response to drought stress during plant development (Chen et al., 2015). GbMYBFL, involving in flavonoid biosynthesis, is most closely related to R2R3-MYB 
and displays high similarity to MYB from other plants (Zhang et al., 2017).

Medicago truncatula is a model plant for genetic research of legume plants (Young and Udvardi, 2009), as it has a small genome, high genetic transformation efficiency, self-pollination, nitrogen fixing, along with high biological diversity. Crop improvement of legume breeding has become an important subject (Lee et al., 2017). MtERF and MtMAPKKK gene families of $M$. truncatula are valuable for characterizing molecular function to improve stress tolerance in plants (Li et al., 2016; Shu et al.. 2016). However, the R2R3-MYB family is poorly identified on a genomic level in $M$. truncatula.

In this study, we performed a genome-wide analysis of the R2R3-MYB gene family in M. truncatula, including multiple alignment analysis, phylogenetic analysis, chromosome localization, gene duplication analysis, and expression profiling. We used quantitative real-time reverse transcription (qRT-PCR) experiments to compare and analyze five stress treatments (ABA, cold, freezing, drought, and salt) with a control treatment. Our study will serve as a foundation for future research into the molecular function of M. truncatula R2R3-MYB genes.

\section{Materials and Methods}

\section{Plant growth and treatment}

M. truncatula (cv. JemalongA17) seeds were grown in a growth chamber (Conviron E15), between $18^{\circ} \mathrm{C}$ (night) and $24^{\circ} \mathrm{C}$ (day), with humidity ranging from $60 \%$ to $80 \%$, and a 14/10 h light-dark period (light, 06:00-20:00). The seedlings were irrigated with half-strength Hoagland solution once every other day. At 8 weeks, M. truncatula were randomly divided into six groups for stress treatments. Plants treated with cold stress (B group) and freezing stress (C group) were transferred to chambers set at $4{ }^{\circ} \mathrm{C}$ and -8 ${ }^{\circ} \mathrm{C}$, respectively. Plants treated with drought stress (D group) and salt stress (E group) were respectively treated with $300 \mathrm{mM}$ mannitol and $200 \mathrm{mM} \mathrm{NaCl}$ solutions. Plants in the ABA (F group) treatment group were sprayed with $100 \mu \mathrm{M}$ ABA solution. The control (untreated, A group) and treated (B-F groups) seedlings were harvested $3 \mathrm{~h}$ after treatment. For each group, five randomly chosen whole seedlings were pooled to form a biological replicate. All plant samples were frozen in liquid nitrogen and stored at $-80{ }^{\circ} \mathrm{C}$ until use.

\section{Database search for MYB proteins in M. truncatula}

$M$. truncatula genome and protein sequences were downloaded from JCVI (http://jcvi.org/medicago/, Mt4.0) (Young et al., 2011). R2R3-MYB protein sequences of Arabidopsis were obtained from TAIR (http://www.arabidopsis.org/) (Poole, 2007) and served as queries to search against the $M$. truncatula proteins using the BLASTP program with e-values of 1E-3. All BLAST hits were retrieved and searched using the Hidden Markov Model (HMM) profile of the R2R2-MYB DNA-binding domain (PF000249), which was downloaded from the Pfam website (http://pfam.xfam.org/) (Finn et al., 2014).

\section{Phylogenetic analysis of the $M$. truncatula R2R3-MYB gene family}

Phylogenetic analysis was performed using MEGA (Version 5.0), and constructed using neighbor-joining (NJ) methods. The NJ method used the following parameters: Poisson correction, pair-wise deletion, and 1000 bootstrap analysis for statistical reliability (Tamura et al., 2011).

\section{Chromosomal location and gene duplication of R2R3-MYB gene family}

The location of R2R3-MYB genes were mapped to different chromosomes using the Circos software (http://circos.ca/) (Krzywinski et al., 2009). If two genes with similarities of more than $85 \%$ were separated by four or fewer gene loci, they were identified as tandem duplications (TD). Otherwise, they were classified as segmental duplications (SD). The duplications with R2R3-MYB genes were identified by plant genome duplications (PGDD, http://chibba.agtec.uga.edu/duplication/) (Lee et al., 2012, Shu et al., 2016), and duplicated genes between different chromosomes or loci were linked in the diagrams.

Expression analysis of R2R3-MYB genes in plant growth and development using high throughput data

We studied the expression analysis of R2R3-MYB genes during growth and development and under different abiotic stress treatments. Gene expression data were downloaded from the Medicago truncatula Gene Expression Atlas (MtGEA) Project (MtGEA, http://mtgea.noble.org/v3/) (Benedito et al., 2008). Genome-wide transcriptome data from $M$. truncatula in different tissues during development were downloaded from the NCBI short read archive database (SRA database) (http://www.ncbi.nlm.nih.gov).

\section{Expression analysis of R2R3-MYB genes under different abiotic stress treatment conditions}

Under six different abiotic stress treatments, MtMYB gene expressional values were evaluated using the TopHat (Trapnell et al., 2009) and Cufflinks (Trapnell et al., 2012) software. The data were analyzed, clustered, and displayed using the ggplot2 of R software (Version 3.1.0), as our previous research described (Shu et al., 2016).

\section{Quantitative reverse transcription PCR (qRT-PCR) analysis of R2R3-MYB genes expressed in $M$. truncatula}

Ten R2R3-MYB genes were selected and quantitative primers were designed based on genes sequences, while the ACTIN and GAPDH genes served as reference genes (see Table S1). Total RNA was extracted from $M$. 
truncatula grown under the six conditions (control, ABA, drought, salt, cold, and freezing) using a total RNA kit (Tiangen, Beijing, China) according to the manufacturer's instructions. The RNA extracts were reverse transcribed to cDNA, using a PrimeScript RT reagent Kit (Toyobo, Shanghai, China). qRT-PCR was performed using the LightCycler ${ }^{\circledR} 96$ System (Roche, Rotkreuz, Switzerland) with SYBR Premix Ex TaqTM II (Toyobo, Shanghai, China). The experiments were repeated for three biological replicates and the PCR conditions were set as follows: $95^{\circ} \mathrm{C}$ for 2 min, 40 cycles of $95^{\circ} \mathrm{C}$ for $30 \mathrm{~s}, 55^{\circ} \mathrm{C}$ for $30 \mathrm{~s}$, and $72^{\circ}$ for $1 \mathrm{~min}$. The fold change value was calculated using the expression abundances, which based on the $2^{-} \Delta \Delta \mathrm{CT}$ method.

\section{Results}

\section{Identification and classification of R2R3-MYB genes in M. truncatula}

To identify R2R3-MYB genes in M. truncatula, Arabidopsis R2R3-MYB gene sequences were used as queries with e-value set as 1E-3. The gene name, gene locus, chromosome location, amino acid sequence, introns, family groups, and isoelectric point (pI) are described in Table 1. Out of the 150 MtMYB genes, 139 genes were distributed across 8 chromosomes of $M$. truncatula. The remaining 11 MtMYB genes were MtMYB5, 6, 7, 13, 14, 16, 34, 35, 36, 37 , and 52. The length of these 139 R2R3-MYB ranged from 194 to 1514 amino acids, with an intron distribution of $0-12$, and the isoelectric point were distributed from 4.77 to 9.93 .

\section{Phylogenetic analysis of the R2R3-MYB genes in $M$. truncatula}

To investigate the evolutionary relationships of R2R3-MYB genes, we performed multiple sequence alignment and phylogenetic analysis. MtMYB transcription factors were queried against AtMYB transcription factors using BLASTP, and we constructed phylogenetic trees of the MtMYB transcription factor family using ClustalW2 and MEGA. MtMYB genes were divided into 21 subfamilies (Figure 1) consistent with the distribution of AtMYB gene family members. The subfamily 1 has six members, subfamily 6 has 14 members, subfamily 13 has seven members, subfamily 14 has ten members, and subfamily 18 has eleven members. This is similar to the distribution of the Arabidopsis subfamily members. R2R3-MYB transcription factors appeared to be conserved in plants as the evolution of M. truncatula is present in the evolution of Arabidopsis.

\section{Chromosomal location and gene duplication of R2R3-MYB gene family}

To determine the evolution and expansion of MYB genes, we used Circos software to construct the distribution of MYB genes across chromosomes (Figure 2). Out of the
150 R2R3-MYB genes, 139 were distributed across 8 chromosomes, mainly chromosome 1, 4, 5, and 7 (MtChr1, MtChr4, MtChr5 and MtChr7, respectively). There were 28 R2R3-MYB genes located on MtChr5 alone. The fewest number of genes were found on MtChr6 with ten R2R3MYB genes. Using sequence alignment, mainly through gene duplication, 128 out of 139 genes were duplicated and divided into two categories. There were 68 segment duplications (SD) caused by the amplification of R2R3-MYB transcription factor members on different chromosomes and 60 tandem duplications (TD) resulting from the generation of R2R3-MYB transcription factor gene clusters. The $\mathrm{SD}$ and TD genes were mainly found on MtChr4, MtChr5, MtChr6, MtChr7, and MtChr8, while other chromosomes only contained SD genes. The regions containing TD genes were hot regions of gene distribution. These duplications may have led to the expansion of the MtMYB gene family in the M. truncatula genome.

\section{Expression analysis of R2R3-MYB genes in growth and development}

The expression information of 71 R2R3-MYB transcription factors were extracted and analyzed by cluster analysis (Figure 3 and Table S2) using the annotation information of microarray data based on MtGEA. We clustered the 71 R2R3-MYB transcription factors into four groups (A-D). Group A was mainly concentrated in the roots and nodules of $M$. truncatula. There was only low expression in other tissues and during developmental processes. Group B was mainly expressed in the development of seed. Group C genes were expressed in various organ tissues. These results indicated that they were both involved in tissues construction process of $M$. truncatula. Finally, Group D genes were expressed at a low level in various tissues and during developmental processes.

The transcriptome sequencing data of $M$. truncatula were downloaded from the NCBI SRA database, and the gene expression level was obtained using TopHat2 and Cufflinks analyses. We extracted 67 R2R3-MYB transcription factors (Figure 4 and Table S3). Multiple genes with high expression in the roots and nodules were classified into Group E, while Group F contained numbers of paralog genes, which were mainly up-regulated in the flower, the carps, and inflorescence.

\section{Expression analysis of R2R3-MYB genes under different abiotic stress treatment conditions}

RNA-seq was used to investigate the expression of R2R3-MYB transcription factors in response to abiotic stress of $M$. truncatula. Out of the 150 MtMYB genes, 64 were expressed in response to abiotic stress (cold, freezing, drought, salt and ABA) (Figure 5 and Table S4). In the control treatment, multiple genes were down-regulated or showed no change in expression, yet few genes were upregulated. We compared genes regulated in response to 
Table 1 - List of all MtMYB genes identified in the Medicago truncatula genome.

\begin{tabular}{|c|c|c|c|c|c|c|}
\hline Gene Name & Gene Locus & Chromosome Location & $\mathrm{AA}$ & Introns & Family Group & $\mathrm{pI}$ \\
\hline MtMYB001 & Medtr3g039990 & chr3:13992780-13994617 & 312 & 2 & 1 & 5.44 \\
\hline MtMYB002 & Medtr6g012180 & chr6:3615718-3617850 & 319 & 2 & 1 & 5.76 \\
\hline MtMYB003 & Medtr7g010210 & chr7:2460324-2462646 & 272 & 3 & 1 & 8.94 \\
\hline MtMYB004 & Medtr7g087130 & chr7:33927709-33929377 & 339 & 2 & 1 & 6.54 \\
\hline MtMYB005 & Medtr0140s0030 & scaffold0140:9682-11671 & 335 & 2 & 1 & 6.6 \\
\hline MtMYB006 & Medtr0489s0020 & scaffold0489:9594-10964 & 335 & 2 & 1 & 6.6 \\
\hline MtMYB007 & Medtr0251s0050 & scaffold0251:9249-10577 & 273 & 1 & 2 & 5.09 \\
\hline MtMYB008 & Medtr1g043050 & $\operatorname{chr} 1: 16126632-16128228$ & 255 & 2 & 2 & 5.58 \\
\hline MtMYB009 & Medtr1g043080 & chr1:16137369-16139502 & 256 & 2 & 2 & 5.6 \\
\hline MtMYB010 & Medtr1g076150 & chr1:33730166-33733155 & 259 & 2 & 2 & 5.31 \\
\hline MtMYB011 & Medtr7g096930 & chr7:38915313-38918018 & 279 & 2 & 2 & 5.35 \\
\hline MtMYB012 & Medtr7g115650 & chr7:47812651-47813895 & 208 & 2 & 2 & 8.51 \\
\hline MtMYB013 & Medtr0008s0390 & scaffold0008:236494-237588 & 273 & 1 & 2 & 5.55 \\
\hline MtMYB014 & Medtr0008s0470 & scaffold0008:261364-262450 & 292 & 1 & 2 & 5.34 \\
\hline MtMYB015 & Medtr2g067420 & chr2:28203900-28207822 & 330 & 2 & 3 & 4.74 \\
\hline MtMYB016 & Medtr0063s0090 & scaffold0063:54059-56064 & 224 & 2 & 3 & 5.78 \\
\hline MtMYB017 & Medtr4g073420 & chr4:27807701-27809440 & 286 & 1 & 4 & 8.65 \\
\hline MtMYB018 & Medtr4g485530 & chr4:33337240-33340075 & 194 & 2 & 4 & 9.68 \\
\hline MtMYB019 & Medtr5g079670 & chr5:34066783-34068495 & 214 & 2 & 4 & 9.14 \\
\hline MtMYB020 & Medtr8g095390 & chr8:39916215-39917940 & 316 & 2 & 4 & 9.14 \\
\hline MtMYB021 & Medtr4g100720 & chr4:41544004-41544940 & 176 & 3 & 5 & 8.25 \\
\hline MtMYB022 & Medtr4g125520 & chr4:52070924-52072242 & 308 & 2 & 5 & 8.34 \\
\hline MtMYB023 & Medtr8g020490 & chr8:7194946-7196540 & 255 & 2 & 5 & 8.55 \\
\hline MtMYB024 & Medtr5g078800 & chr5:33679342-33680505 & 266 & 2 & 6 & 8.76 \\
\hline MtMYB025 & Medtr5g078860 & chr5:33697936-33699039 & 307 & 2 & 6 & 8.76 \\
\hline MtMYB026 & Medtr5g078910 & chr5:33733725-33736557 & 301 & 2 & 6 & 9.06 \\
\hline MtMYB027 & Medtr5g078930 & chr5:33748446-33750303 & 220 & 2 & 6 & 9.74 \\
\hline MtMYB028 & Medtr5g078950 & chr5:33762292-33763827 & 266 & 2 & 6 & 9.14 \\
\hline MtMYB029 & Medtr5g079120 & chr5:33821199-33824299 & 307 & 3 & 6 & 9.12 \\
\hline MtMYB030 & Medtr5g079220 & chr5:33865422-33867467 & 277 & 2 & 6 & 8.08 \\
\hline MtMYB031 & Medtr5g079290 & chr5:33903955-33904999 & 265 & 2 & 6 & 9.11 \\
\hline MtMYB032 & Medtr7g017260 & chr7:5468456-5471716 & 276 & 2 & 6 & 7.59 \\
\hline MtMYB033 & Medtr8g060940 & chr8:21305461-21306435 & 245 & 2 & 6 & 5.83 \\
\hline MtMYB034 & Medtr0001s0360 & scaffold0001:148343-149371 & 254 & 2 & 6 & 8.13 \\
\hline MtMYB035 & Medtr0193s0090 & scaffold0193:23279-25596 & 208 & 2 & 6 & 9.27 \\
\hline MtMYB036 & Medtr0197s0010 & scaffold0197:3158-6293 & 230 & 2 & 6 & 5.79 \\
\hline MtMYB037 & Medtr0247s0040 & scaffold0247:19831-22448 & 180 & 2 & 6 & 9.56 \\
\hline MtMYB038 & Medtr2g034790 & chr2:13342703-13345610 & 333 & 2 & 7 & 5.57 \\
\hline MtMYB039 & Medtr4g121460 & chr4:50199327-50201231 & 340 & 2 & 7 & 5.21 \\
\hline MtMYB040 & Medtr7g117730 & chr7:48891947-48893156 & 257 & 1 & 7 & 9.19 \\
\hline MtMYB041 & Medtr8g027345 & chr8:9621235-9622899 & 301 & 1 & 7 & 7.03 \\
\hline MtMYB042 & Medtr1g100653 & chr1:45593395-45595446 & 296 & 2 & 9 & 7.16 \\
\hline MtMYB043 & Medtr4g082230 & chr4:31939369-31942857 & 408 & 2 & 9 & 6.02 \\
\hline MtMYB044 & Medtr4g082290 & chr4:31999665-32001816 & 408 & 2 & 9 & 5.77 \\
\hline MtMYB045 & Medtr6g012690 & chr6:3910120-3912657 & 358 & 2 & 9 & 5.54 \\
\hline MtMYB046 & Medtr7g011170 & chr7:2948411-2950937 & 310 & 2 & 9 & 6.57 \\
\hline MtMYB047 & Medtr7g076740 & chr7:28944308-28947368 & 305 & 2 & 9 & 8.15 \\
\hline MtMYB048 & Medtr8g031360 & chr8:11745382-11748030 & 298 & 2 & 9 & 5.92 \\
\hline MtMYB049 & Medtr4g478180 & chr4:30006885-30009067 & 354 & 3 & 10 & 6.36 \\
\hline
\end{tabular}




\begin{tabular}{|c|c|c|c|c|c|c|}
\hline MtMYB050 & Medtr5g007370 & chr5:1317895-1319526 & 300 & 1 & 10 & 6.25 \\
\hline MtMYB051 & Medtr5g029840 & chr5:12551795-12553088 & 335 & 1 & 10 & 6.26 \\
\hline MtMYB052 & Medtr0008s 0280 & scaffold0008:117749-119885 & 232 & 2 & 10 & 6.21 \\
\hline MtMYB053 & Medtr2g011660 & chr2:2891380-2892991 & 346 & 2 & 11 & 5.85 \\
\hline MtMYB054 & Medtr2g089450 & chr2:37807548-37809745 & 251 & 2 & 11 & 7.62 \\
\hline MtMYB055 & Medtr2g089620 & chr2:37880385-37882262 & 327 & 2 & 11 & 6.45 \\
\hline MtMYB056 & Medtr4g091490 & chr4:36245993-36247326 & 359 & 2 & 11 & 5.87 \\
\hline MtMYB057 & Medtr1g085040 & chr1:37962367-37964302 & 370 & 2 & 13 & 7.1 \\
\hline MtMYB058 & Medtr1g085640 & chr1:38268101-38269978 & 389 & 2 & 13 & 6.1 \\
\hline MtMYB059 & Medtr1g112760 & $\operatorname{chr} 1: 51044088-51045093$ & 231 & 2 & 13 & 8.61 \\
\hline MtMYB060 & Medtr3g077110 & chr3:34625609-34627040 & 350 & 2 & 13 & 5.71 \\
\hline MtMYB061 & Medtr4g105130 & chr4:43559175-43561153 & 362 & 2 & 13 & 5.51 \\
\hline MtMYB062 & Medtr7g110830 & chr7:45437545-45439504 & 368 & 2 & 13 & 5.28 \\
\hline MtMYB063 & Medtr7g111290 & chr7:45674622-45676610 & 382 & 2 & 13 & 6.26 \\
\hline MtMYB064 & Medtr1g017000 & chr1:4609270-4612231 & 253 & 2 & 14 & 6.22 \\
\hline MtMYB065 & Medtr1g017140 & chr1:4685126-4687287 & 342 & 2 & 14 & 7.21 \\
\hline MtMYB066 & Medtr2g095520 & chr2:40818577-40820355 & 321 & 2 & 14 & 7.02 \\
\hline MtMYB067 & Medtr3g103570 & chr3:47845667-47847265 & 348 & 2 & 14 & 6.26 \\
\hline MtMYB068 & Medtr4g057635 & chr4:21173419-21175385 & 325 & 2 & 14 & 6.67 \\
\hline MtMYB069 & Medtr4g097570 & chr4:40253351-40254860 & 294 & 2 & 14 & 8.04 \\
\hline MtMYB070 & Medtr4g128670 & chr4:53556826-53558636 & 351 & 2 & 14 & 5.43 \\
\hline MtMYB071 & Medtr5g014990 & chr5:5113035-5114677 & 310 & 2 & 14 & 7.49 \\
\hline MtMYB072 & Medtr6g090405 & chr6:34357171-34358762 & 256 & 2 & 14 & 5.53 \\
\hline MtMYB073 & Medtr7g102110 & chr7:41207062-41209098 & 273 & 2 & 14 & 6.13 \\
\hline MtMYB074 & Medtr1g100667 & chr1:45604161-45606367 & 273 & 2 & 16 & 9.4 \\
\hline MtMYB075 & Medtr6g055910 & chr6:20067285-20071069 & 284 & 2 & 16 & 6.55 \\
\hline MtMYB076 & Medtr1g083630 & chr1:37214098-37220601 & 631 & 10 & 18 & 8.87 \\
\hline MtMYB077 & Medtr1g085770 & chr1:38342281-38444471 & 308 & 3 & 18 & 7.64 \\
\hline MtMYB078 & Medtr1g085880 & chr1:38446474-38447221 & 194 & 1 & 18 & 9.25 \\
\hline MtMYB079 & Medtr2g088170 & chr2:37135155-37138193 & 460 & 2 & 18 & 5.04 \\
\hline MtMYB080 & Medtr5g062790 & chr5:26032596-26033729 & 305 & 2 & 18 & 4.67 \\
\hline MtMYB081 & Medtr5g088080 & chr5:38180518-38181517 & 267 & 2 & 18 & 5.06 \\
\hline MtMYB082 & Medtr5g088150 & chr5:38189614-38190613 & 267 & 2 & 18 & 5.06 \\
\hline MtMYB083 & Medtr5g088610 & chr5:38446993-38448346 & 356 & 2 & 18 & 5.03 \\
\hline MtMYB084 & Medtr5g088640 & chr5:38455878-38456905 & 276 & 2 & 18 & 5.06 \\
\hline MtMYB085 & Medtr5g488170 & chr5:38207742-38208675 & 245 & 2 & 18 & 8.27 \\
\hline MtMYB086 & Medtr5g488210 & chr5:38216839-38217838 & 267 & 2 & 18 & 5.06 \\
\hline MtMYB087 & Medtr6g006030 & chr6:968935-973160 & 210 & 2 & 19 & 6.79 \\
\hline MtMYB088 & Medtr7g035075 & chr7:13292852-13298285 & 209 & 2 & 19 & 6.07 \\
\hline MtMYB089 & Medtr1g086510 & $\operatorname{chr} 1: 38706430-38707982$ & 266 & 2 & 20 & 6.71 \\
\hline MtMYB090 & Medtr1g086530 & $\operatorname{chr} 1: 38719605-38720569$ & 279 & 1 & 20 & 5.57 \\
\hline MtMYB091 & Medtr1g110460 & chr1:49847994-49849320 & 312 & 2 & 20 & 6.97 \\
\hline MtMYB092 & Medtr2g033170 & chr2:12558787-12561118 & 330 & 2 & 20 & 5.54 \\
\hline MtMYB093 & Medtr4g123040 & chr4:50776703-50778712 & 316 & 2 & 20 & 6.9 \\
\hline MtMYB094 & Medtr4g082040 & chr4:31789875-31791702 & 428 & 2 & 21 & 5.48 \\
\hline MtMYB095 & Medtr5g041570 & chr5:18244022-18246575 & 439 & 2 & 21 & 5.81 \\
\hline MtMYB096 & Medtr6g027340 & chr6:9347619-9348708 & 206 & 2 & 21 & 9.43 \\
\hline MtMYB097 & Medtr6g027370 & chr6:9359727-9361707 & 353 & 2 & 21 & 9.13 \\
\hline MtMYB098 & Medtr6g074860 & chr6:27791872-27795423 & 242 & 2 & 21 & 8.94 \\
\hline MtMYB099 & Medtr7g086960 & chr7:33824449-33826853 & 357 & 2 & 21 & 8.87 \\
\hline MtMYB100 & Medtr3g101290 & chr3:46598679-46599616 & 227 & 0 & 22 & 6.39 \\
\hline
\end{tabular}




\begin{tabular}{|c|c|c|c|c|c|c|}
\hline MtMYB101 & Medtr4g094982 & chr4:39426938-39428438 & 272 & 0 & 22 & 5.76 \\
\hline MtMYB102 & Medtr5g016510 & chr5:5923364-5924314 & 317 & 0 & 22 & 8.35 \\
\hline MtMYB103 & Medtr5g082910 & chr5:35747490-35748793 & 289 & 1 & 22 & 8.33 \\
\hline MtMYB104 & Medtr6g015455 & chr6:5126272-5138191 & 1514 & 9 & 22 & 6.6 \\
\hline MtMYB105 & Medtr4g019370 & chr4:6040082-6046125 & 351 & 1 & 23 & 6.1 \\
\hline MtMYB106 & Medtr3g074520 & chr3:33695057-33696802 & 347 & 2 & 24 & 5.76 \\
\hline MtMYB107 & Medtr1g026870 & chr1:8820780-8826887 & 984 & 10 & 25 & 5.45 \\
\hline MtMYB108 & Medtr1g045610 & chr1:17117606-17119422 & 259 & 2 & 25 & 9.28 \\
\hline MtMYB109 & Medtr1g057980 & chr1:25521596-25523733 & 298 & 2 & 25 & 6.68 \\
\hline MtMYB110 & Medtr1g062940 & $\operatorname{chr} 1: 27632125-27633748$ & 219 & 3 & 25 & 5.7 \\
\hline MtMYB111 & Medtr1g073170 & chr1:32447073-32448262 & 258 & 2 & 25 & 7.64 \\
\hline MtMYB112 & Medtr2g064160 & chr2:27171680-27173646 & 314 & 2 & 25 & 6.14 \\
\hline MtMYB113 & Medtr2g096380 & chr2:41173925-41175393 & 237 & 2 & 25 & 6.2 \\
\hline MtMYB114 & Medtr2g097910 & chr2:41830130-41832026 & 320 & 1 & 25 & 5.63 \\
\hline MtMYB115 & Medtr2g099740 & chr2:42787607-42789410 & 402 & 1 & 25 & 6.27 \\
\hline MtMYB116 & Medtr3g065440 & chr3:29570010-29573532 & 312 & 3 & 25 & 4.81 \\
\hline MtMYB117 & Medtr3g077650 & chr3:34915543-34917386 & 448 & 2 & 25 & 6.65 \\
\hline MtMYB118 & Medtr3g083540 & chr3:37705148-37706353 & 304 & 1 & 25 & 6.83 \\
\hline MtMYB119 & Medtr3g097450 & chr3:44673726-44675570 & 298 & 2 & 25 & 8.71 \\
\hline MtMYB120 & Medtr3g110028 & chr3:50889343-50895792 & 904 & 11 & 25 & 5.08 \\
\hline MtMYB121 & Medtr3g461490 & chr3:24436965-24438138 & 326 & 2 & 25 & 6.31 \\
\hline MtMYB122 & Medtr4g063100 & chr4:23343932-23345888 & 254 & 2 & 25 & 5.44 \\
\hline MtMYB123 & Medtr4g065017 & chr4:24357511-24360058 & 333 & 1 & 25 & 5.31 \\
\hline MtMYB124 & Medtr4g088015 & chr4:34653348-34657396 & 443 & 2 & 25 & 9.15 \\
\hline MtMYB125 & Medtr4g102380 & chr4:42421826-42423447 & 273 & 1 & 25 & 5.26 \\
\hline MtMYB126 & Medtr4g105660 & chr4:43865250-43867027 & 431 & 2 & 25 & 6.39 \\
\hline MtMYB127 & Medtr5g010020 & chr5:2590454-2592700 & 340 & 2 & 25 & 5.8 \\
\hline MtMYB128 & Medtr5g010650 & chr5:2899487-2902910 & 477 & 7 & 25 & 8.32 \\
\hline MtMYB129 & Medtr5g038910 & chr5:17123381-17129369 & 391 & 10 & 25 & 6.19 \\
\hline MtMYB130 & Medtr5g049190 & chr5:21549233-21551259 & 243 & 2 & 25 & 6.36 \\
\hline MtMYB131 & Medtr5g070020 & chr5:29670807-29672960 & 356 & 2 & 25 & 8.58 \\
\hline MtMYB132 & Medtr5g078140 & chr5:33378172-33380732 & 321 & 2 & 25 & 5.12 \\
\hline MtMYB133 & Medtr6g009430 & chr6:2790201-2792486 & 337 & 2 & 25 & 5.59 \\
\hline MtMYB134 & Medtr7g035350 & chr7:13467452-13470094 & 451 & 2 & 25 & 5.66 \\
\hline MtMYB135 & Medtr7g037130 & chr7:13641861-13644153 & 359 & 2 & 25 & 8.62 \\
\hline MtMYB136 & Medtr7g037260 & chr7:13704241-13706602 & 359 & 2 & 25 & 8.99 \\
\hline MtMYB137 & Medtr7g061330 & chr7:22157000-22164437 & 531 & 7 & 25 & 9.15 \\
\hline MtMYB138 & Medtr7g061550 & chr7:22184367-22187445 & 360 & 1 & 25 & 9.39 \\
\hline MtMYB139 & Medtr7g450950 & chr7:17066918-17069310 & 339 & 2 & 25 & 5.53 \\
\hline MtMYB140 & Medtr7g451170 & chr7:17173579-17176966 & 413 & 2 & 25 & 9.22 \\
\hline MtMYB141 & Medtr7g461410 & chr7:22216202-22220641 & 567 & 6 & 25 & 8.75 \\
\hline MtMYB142 & Medtr8g006470 & chr8:571945-573793 & 288 & 2 & 25 & 6.22 \\
\hline MtMYB143 & Medtr8g017340 & chr8:5841430-5843448 & 493 & 2 & 25 & 4.92 \\
\hline MtMYB144 & Medtr8g017350 & chr8:5847584-5850190 & 644 & 3 & 25 & 4.79 \\
\hline MtMYB145 & Medtr8g017390 & chr8:5885779-5887719 & 476 & 2 & 25 & 5.34 \\
\hline MtMYB146 & Medtr8g017440 & chr8:5919956-5921521 & 342 & 2 & 25 & 6.45 \\
\hline MtMYB147 & Medtr8g017500 & chr8:5958601-5960679 & 349 & 2 & 25 & 5.96 \\
\hline MtMYB148 & Medtr8g017540 & chr8:5983119-5985752 & 395 & 3 & 25 & 6.07 \\
\hline MtMYB149 & Medtr8g098860 & chr8:41349431-41351751 & 321 & 2 & 25 & 5.85 \\
\hline MtMYB150 & Medtr8g468380 & chr8:24758342-24766275 & 437 & 12 & 25 & 8.72 \\
\hline
\end{tabular}




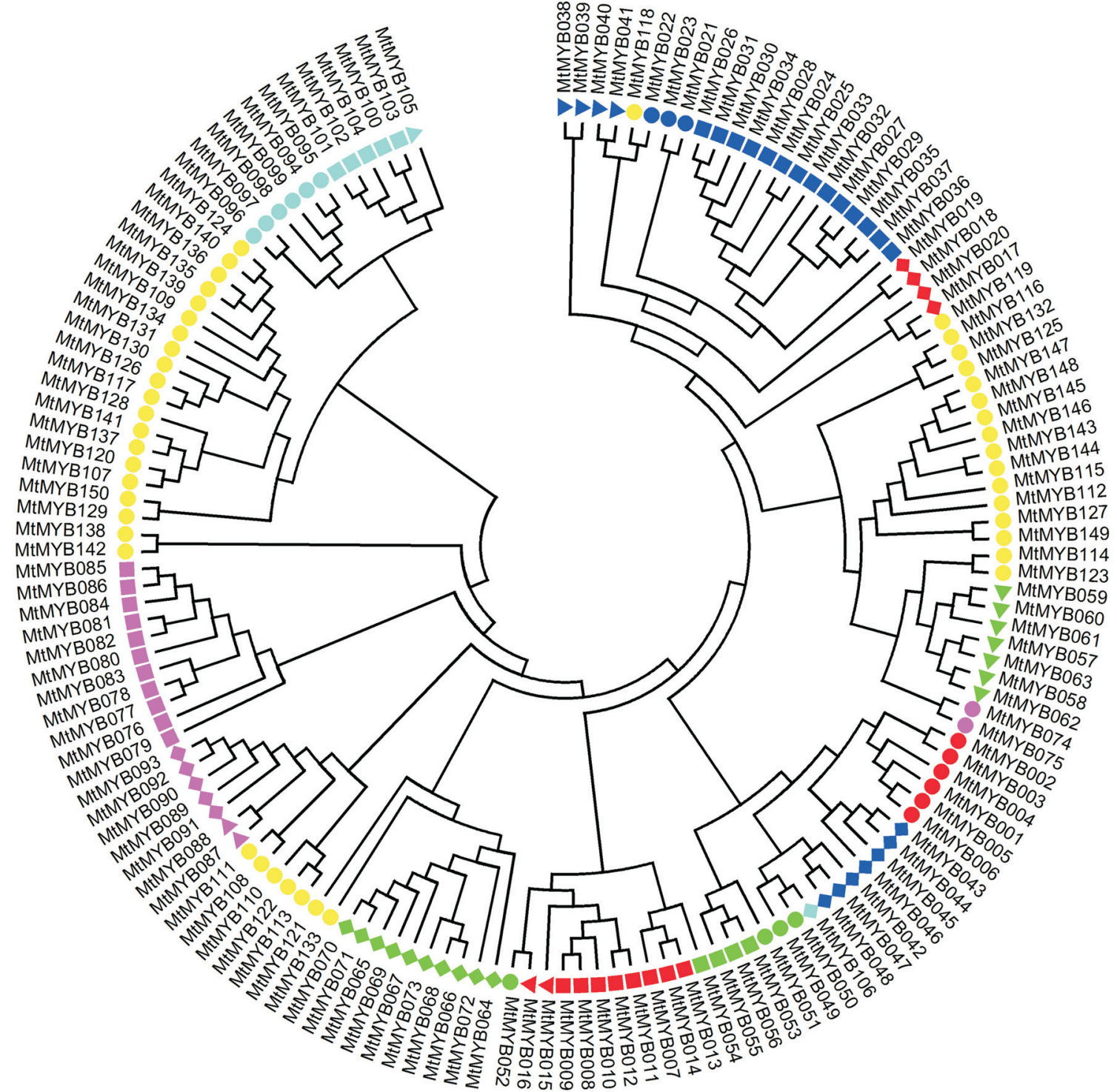

Figure 1 - Phylogenetic tree analysis of the R2R3-MYB transcription factors in Medicago truncatula. Red circle: subgroup 1; red square: subgroup 2; red triangle: subgroup 3; red diamond: subgroup 4; blue circle: subgroup five; blue square subgroup six; blue indicates subgroup seven; blue diamond subgroup nine; green circular subgroup ten; green square subgroup 11; green triangle: subgroup 13; green diamond: subgroup 14; pink circle: subgroup 16; pink square: subgroup 18; pink triangle: subgroup 19; pink diamond: subgroup 20; cyan circle: subgroup 21; cyan square: subgroup 22; cyan triangle: subgroup 23; cyan diamond: subgroup 24; yellow circle: subgroup 25.

abiotic stress to the control treatment and found genes highly up-regulated in response to cold and freezing stresses $(50 / 64,78.1 \%)$. These genes interacted with their paralog genes showing co-expression. In response to drought, high salt, and ABA stresses, multiple genes showed opposing expression and co-expression of the pairs of paralog genes. Significantly, most of these genes showed only low expression or no expression. In particular, some genes were down-regulated and co-expressed in response to ABA stress. It was suggested that the expression of these transcription factors are induced by ABA hormones, which regulate the downstream response genes and affect the response of plants to drought stress and salt stress.
qRT-PCR validation of R2R3-MYB genes expression

To validate the reliability of the RNA-seq data under abiotic stress in M. truncatula, we selected 10 R2R3-MYB transcription factors (MtMYB010, MtMYB011, MtMYB012, MtMYB013, MtMYB014, MtMYB054, MtMYB090, MtMYB100, MtMYB108, and MtMYB116) from $M$. truncatula for qRT-PCR validation. The expression levels of the RNA-seq data and qRT-PCR gene expression analysis resulted in a correlation coefficient of 0.85 (Figure 6). These results suggest the RNA-seq data are highly reliable. 


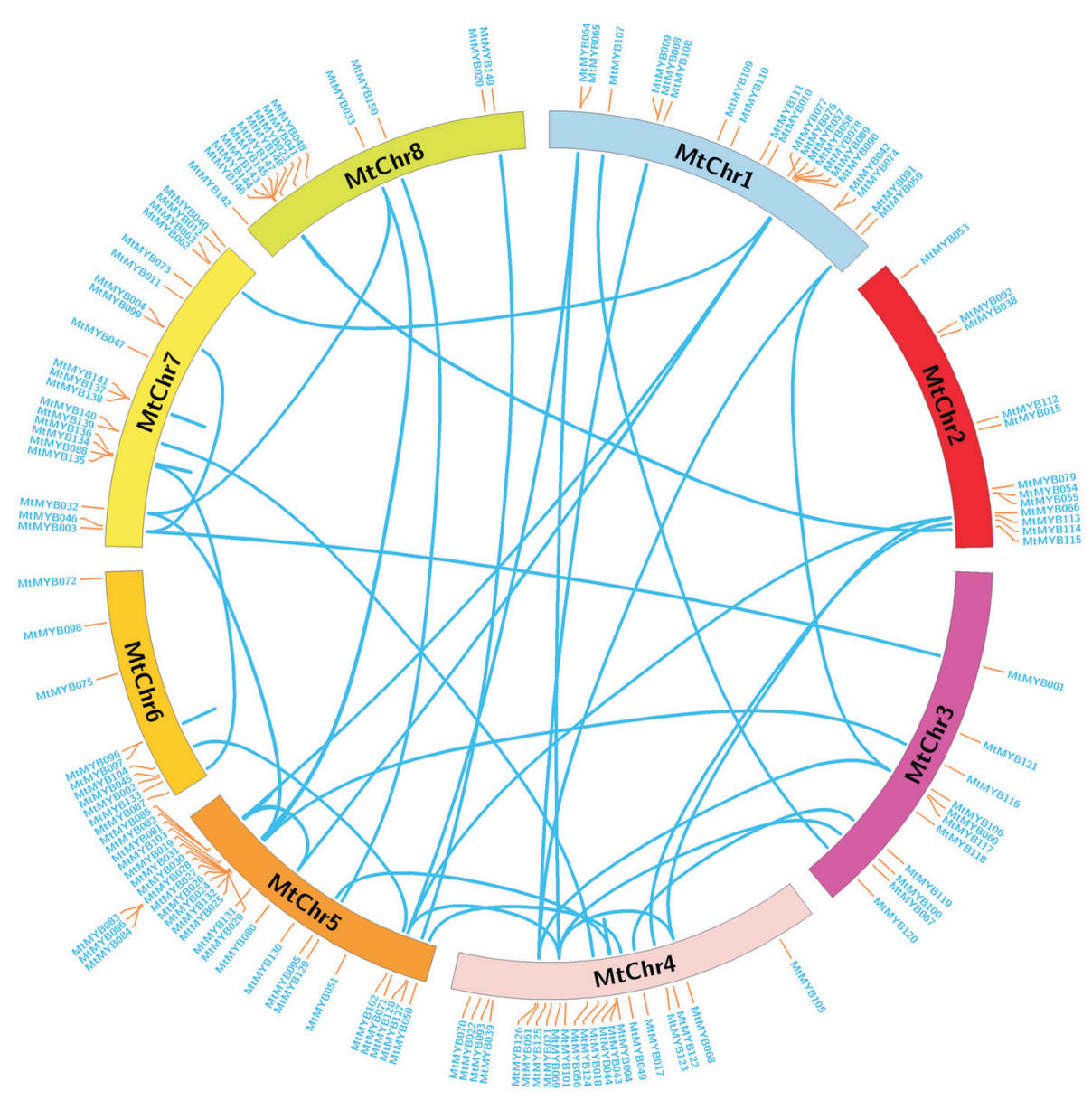

Figure 2 - Chromosome distribution and expansion analysis of R2R3-MYB transcription factors in Medicago truncatula. The genome locations of R2R3-MYB transcription factors were retrieved from Medicago genome website, and the duplications between R2R3-MYB genes identified using software PGDD and BLAST analysis.

\section{Discussion}

To date, R2R3-MYB transcription factors have been identified in plants, including Arabidopsis (126) (Yanhui et al., 2006), maize (157) (Du et al., 2012a), rice (102) (Yanhui et al., 2006), Populus (192) (Wilkins et al., 2009), and cassava (166) (Liao et al., 2016). The M. truncatula genome has been sequenced, yet the R2R3-MYB transcription factors have not been researched. This study performed genome-wide analysis of the R2R3-MYB transcription factors in M. truncatula. We identified 150 R2R3-MYB genes in $M$. truncatula and compared these with other plants. The R2R3-MYB genes of $M$. truncatula are similar to their Arabidopsis, maize, and cassava counterparts. The results indicated that R2R3-MYB transcription factors are highly conserved in plants.

Cannon et al. (2004) have shown gene duplication was closely related to plant evolution and played an important role in the gene amplification. Gene duplication analysis showed the same subfamily members were located on different chromosomes, such as the 21 subfamily gene members. These genes were distributed across all chromosomes and occurred as TD and SD. These results were consistent with the results of the phylogenetic tree.
Furthermore, distribution of other subfamily members was confirmed based on clustering in the phylogenetic tree. These results suggest that the R2R3-MYB genes in $M$. truncatula are highly conserved as a result of gene duplication. The duplication pattern of R2R3-MYB genes, are consistent with the expression of R2R3-MYB genes in tomato (Zhao et al., 2014) and Populus (Wilkins et al., 2009). Tandem gene duplication was a major driver of gene expansion in M. truncatula.

MYB genes play a role in plant development and stress tolerance (Martin and Pazares, 1997). We have shown that R2R3-MYB genes were typically expressed in the roots, nodules, seedpods, and flowers. Compared with soybean and Arabidopsis, differential expression was observed during flower development, root formation, and seed development for R2R3-MYB genes (Du et al., 2012b). Nodulation is the result of a symbiosis between legumes and rhizobial bacteria in soil. Libault et al. (2009) have reported a gene named Control of Nodule Development (CND), encoding an MYB transcription factor gene. When the CND gene is silenced, nodulation is reduced (Libault et al., 2009). These results indicate that the MYB 


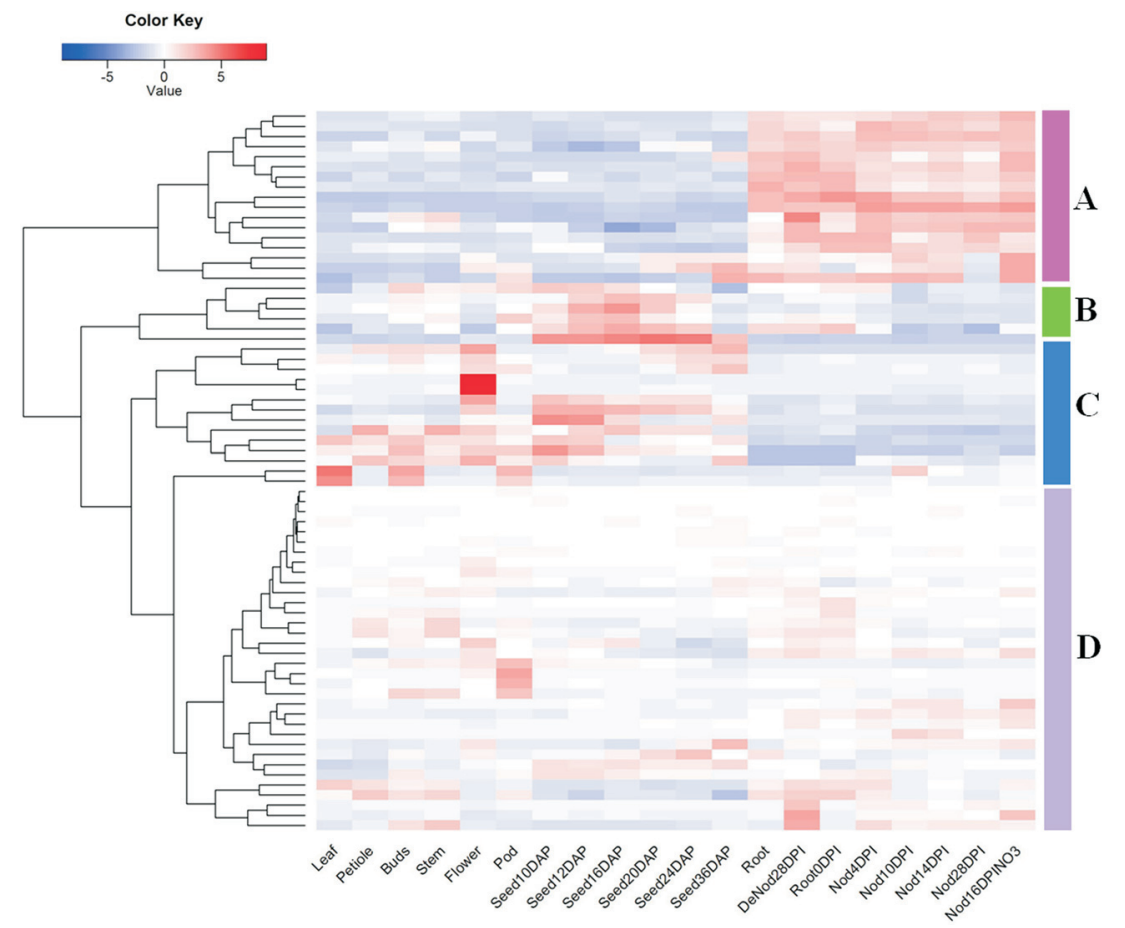

Figure 3 - Microarray expression data of R2R3-MYB transcription factors in Medicago truncatula. The heatmap was generated using R gplots package. The expressional values of 71 R2R3-MYB genes were retrieved from MtGEA, and they were normalized and used as input, red represents high expressional levels, while blue represents low expressional level.

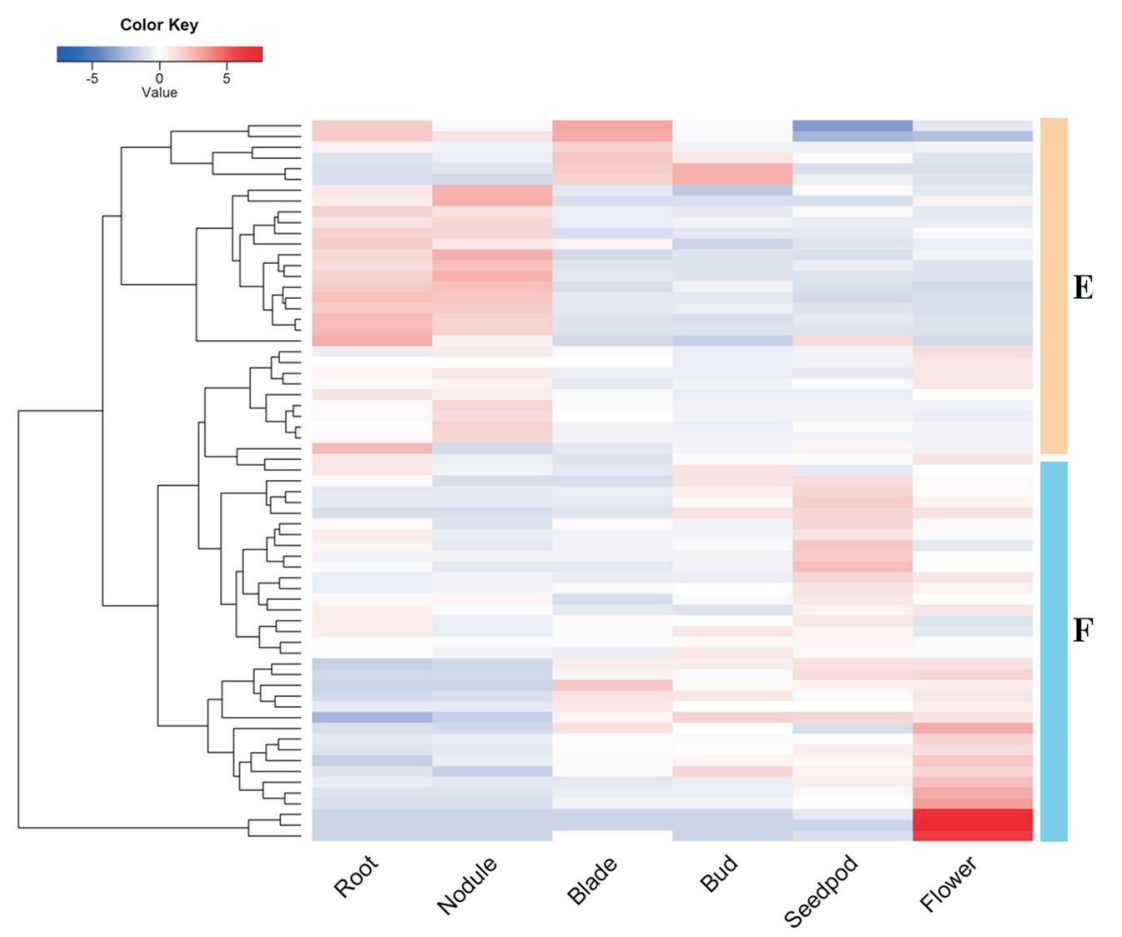

Figure 4 - RNA-seq analysis of R2R3-MYB transcription factors in Medicago truncatula. The heatmap was generated using R gplots package, and the FPKM values of Medicago truncatula genes were evaluated and normalized based RNA-seq data from NCBI SRA database. The plot data included expressional profiles of 67 R2R3-MYB genes in six tissues, and red represents high expressional levels, while blue represents low expressional level. transcription factors may play a major role in regulation of 2013). Their results show that MYB genes are involved in legume-specific nodulation.

MYB transcription factor genes have been investigated as regulators for plant responses (Ambawat et al., response to various abiotic stresses in higher plants. Herein, we found that MYB genes were not only down-regulated, but in some cases up-regulated in response to drought and 


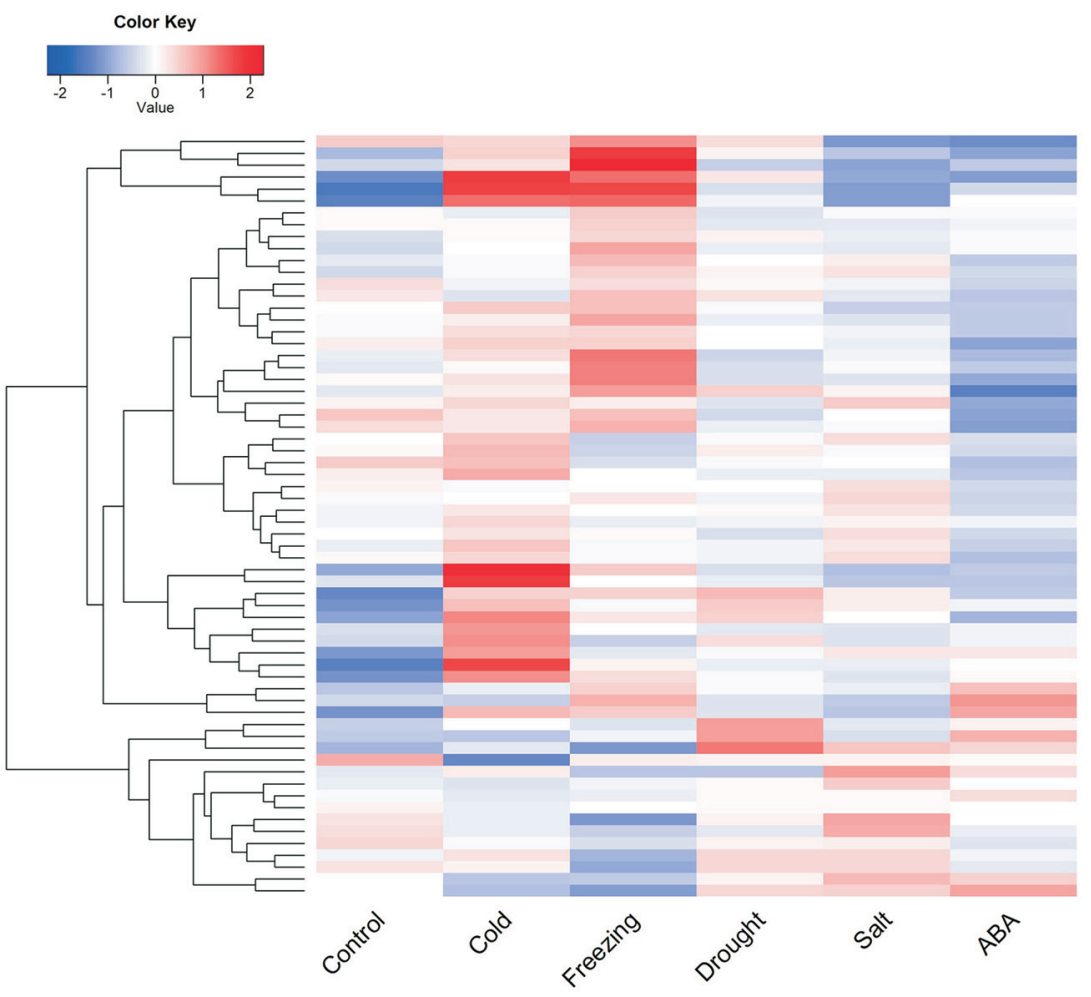

Figure 5 - Expression profile analysis of R2R3-MYB transcription factors in response to abiotic stresses in Medicago truncatula. The heatmap was generated using R gplots package, and the FPKM values of Medicago truncatula genes were evaluated and normalized based RNA-seq data from NCBI SRA database. The plot data included expressional profiles of 64 R2R3-MYB genes in response to abiotic stress, and red represents high expressional levels, while blue represents low expressional level.

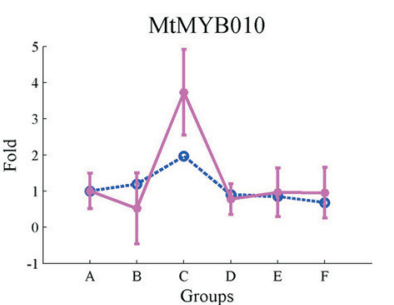

MtMYB014
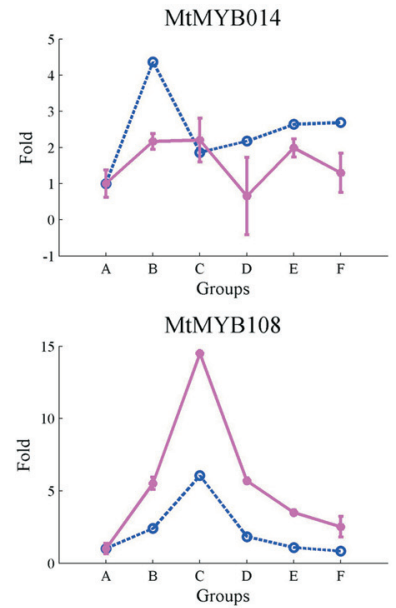

MtMYB011

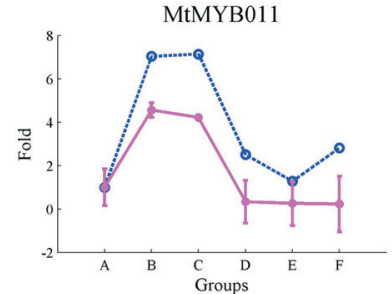

MtMYB054

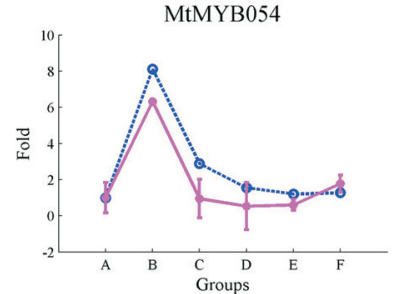

MtMYB116

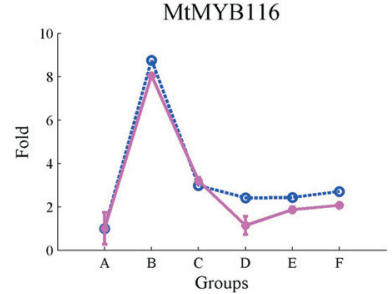

MtMYB012

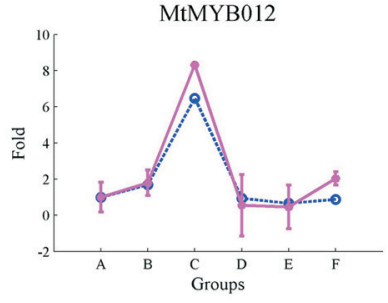

MtMYB090

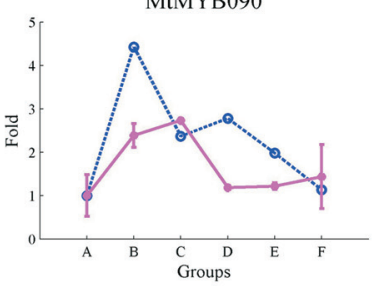

$\stackrel{\cdots \cdots \text { RNA-seq }}{\cdots-\text { qRT-PCR }}$
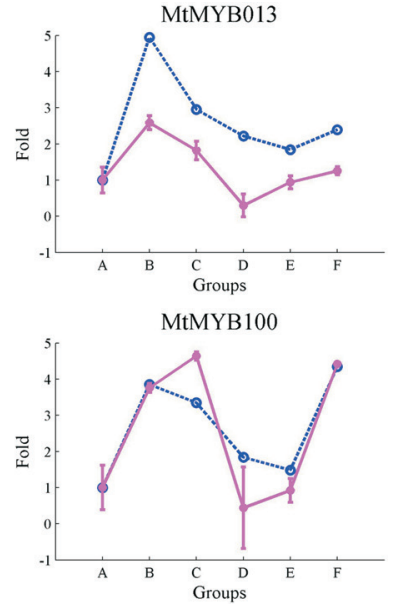

Figure 6 - qRT-PCR validation of R2R3-MYB transcription factors in Medicago truncatula. All expressional levels of each R2R3-MYB genes were normalized with control expression value (group A) set as one. The groups B (cold stress), C (freezing stress), D (drought stress), E (salt stress), and F (ABA treatment) were compared with control group, and fold-changes were calculated. The fold-change data was used to create the plot, and the blue indicates RNA-seq results, while pink indicates qRT-PCR analysis results. 
salt stress. A positive response of MYB genes following drought stress, salt stress, and ABA-induced stress has been observed in Arabidopsis (AtMYB002, AtMYB060/AtMYB094, AtMYB044, and AtMYB096) (Cominelli et al., 2005; Jung et al., 2008; Seo et al., 2009; Urao and Shinozaki, 1993), Boea crassifolia (BcMYB1) (Chen et al., 2005), and Saccharum officinarum (ScMYBAS1) (Prabu and Prasad, 2012). However, MtMYB genes were up-regulated in response to cold and freezing stresses, while the opposite is observed in Arabidopsis. Interestingly, our result agrees with the expression profile of cotton in response to drought and salt stress (He et al., 2016). Both cotton and M. truncatula are diploid and their duplication can be divided into TD and SD. The expression pattern of $M$. truncatula MYB genes under different abiotic stress conditions suggest that some may play a major role in cross-talk among different signal transduction pathways in response to abiotic stresses.

\section{Conclusions}

In summary, we have identified 150 MYB genes in M. truncatula, which were classified into 21 subfamilies based on phylogenetic analysis. Meanwhile, their expression profiles were investigated using microarray and RNA-seq. The results revealed regulatory roles in plant growth and tissue development, and especially nodule development. In addition, we explored the role of the MYB genes in response to abiotic stresses. Our results suggested MYB transcription factors broadly participate in abiotic stress response of $M$. truncatula, whose function can be carefully explored in future.

\section{Acknowledgments}

We thank Miss Jun Zhang and Dr. Lili Song (Harbin Normal University) for their helpful technical supports in plant growth and treatment. The data analysis work was supported by high performance computing center of Harbin Normal University. This work was supported by grants from the National Natural Science Foundation of China (31302019 and 31470571), the China Postdoctoral Science Foundation (2016T90307 and 2015M571430), the University Nursing Program for Young Scholars with Creative Talents in Heilongjiang Province (UNPYST-2016071), the MOST 863 Project (2013AA102607-5), and the Heilongjiang Province Postdoctoral Science Foundation (LBH-Z14126).

\section{Author Contributions}

YS and CG conceived and designed the experiments; WL, YL, JZ, and ZX performed the experiments; YS, WL, JZ, and ZX analyzed the data; YS, WL, and CG wrote the paper. All authors read and approved the final version.

\section{Conflict of Interest}

The authors declare that there is no conflict of interest regarding the publication of this paper.

\section{References}

Al-Attala MN, Wang X, Abou-Attia MA, Duan X and Kang Z (2014) A novel TaMYB4 transcription factor involved in the defence response against Puccinia striiformis f. sp. tritici and abiotic stresses. Plant Mol Biol 84:589-603.

Ambawat S, Sharma P, Yadav NR and Yadav RC (2013) MYB transcription factor genes as regulators for plant responses: an overview. Physiol Mol Biol Plants 19:307-321

Benedito VA, Torres-Jerez I, Murray JD, Andriankaja A, Allen S, Kakar K, Wandrey M, Verdier J, Zuber H, Ott T et al. (2008) A gene expression atlas of the model legume Medicago truncatula. Plant J 55:504-513.

Cannon SB, Mitra A, Baumgarten A, Young ND and May G (2004) The roles of segmental and tandem gene duplication in the evolution of large gene families in Arabidopsis thaliana. BMC Plant Biol 4:10.

Chen BJ, Wang Y, Hu YL, Wu Q and Pin ZP (2005) Cloning and characterization of a drought inducible MYB gene from Boea crassifolia. Plant Sci 168:493-500.

Chen T, Li W, Hu X, Guo J, Liu A and Zhang B (2015) A cotton MYB transcription factor, GbMYB5, is positively involved in plant adaptive response to drought stress. Plant Cell Physiol 56:917-929.

Cominelli E, Galbiati M, Vavasseur A, Conti L, Sala T, Vuylsteke M, Leonhartd N, Dellaporta SL and Tonelli C (2005) A guard-cell-specific MYB transcription factor regulates stomatal movements and plant drought tolerance. Curr Biol 15:1196-1200

Denekamp M and Smeekens SC (2003) Integration of wounding and osmotic stress signals determines the expression of the AtMYB102 transcription factor gene. Plant Physiol 132:1415-1423.

Du H, Feng BR, Yang SS, Huang YB and Tang YX (2012a) The R2R3-MYB transcription factor gene family in maize. PLoS One 7:e37463.

Du H, Yang SS, Liang Z, Feng BR, Liu L, Huang YB and Tang YX (2012b) Genome-wide analysis of the MYB transcription factor superfamily in soybean. BMC Plant Biol 12:106.

Elkereamy A, Bi YM, Ranathunge K, Beatty PH, Good AG and Rothstein SJ (2012) The rice R2R3-MYB transcription factor OsMYB55 is involved in the tolerance to high temperature and modulates amino acid metabolism. PLoS One 7:e52030.

Finn RD, Bateman A, Clements J, Coggill P, Eberhardt RY, Eddy SR, Heger A, Hetherington K, Holm L, Mistry J et al. (2014) Pfam: The protein families database. Nucleic Acids Res 42:D222-D230.

He Q, Jones DC, Li W, Xie F, Ma J, Sun R, Wang Q, Zhu S and Zhang B (2016) Genome-wide identification of R2R3-MYB genes and expression analyses during abiotic stress in Gossypium raimondii. Sci Rep 6:22980.

Jin H and Martin C (1999) Multifunctionality and diversity within the plant MYB-gene family. Plant Mol Biol 41:577-585.

Jung C, Seo JS, Han SW, Koo YJ, Kim CH, Song SI, Nahm BH, Choi YD and Cheong JJ (2008) Overexpression of AtMYB44 enhances stomatal closure to confer abiotic stress 
tolerance in transgenic Arabidopsis. Plant Physiol 146:623-635.

Kanei-Ishii C, Sarai A, Sawazaki T, Nakagoshi H, He DN, Ogata K, Nishimura Y and Ishii S (1990) The tryptophan cluster: A hypothetical structure of the DNA-binding domain of the myb protooncogene product. J Biol Chem 265:19990-19995.

Krzywinski M, Schein J, Birol I, Connors J, Gascoyne R, Horsman D, Jones SJ and Marra MA (2009) Circos: An information aesthetic for comparative genomics. Genome Res 19:1639-1645.

Lee C, Yu D, Choi HK and Kim RW (2017) Reconstruction of a composite comparative map composed of ten legume genomes. Genes Genom 39:111.

Lee TH, Tang H, Wang X and Paterson AH (2012) PGDD: a database of gene and genome duplication in plants. Nucleic Acids Res 41: D1152-D1158.

Li W, Xu H, Liu Y, Song L, Guo C and Shu Y (2016) Bioinformatics analysis of MAPKKK family genes in Medicago truncatula. Genes 7: E13.

Liao W, Yang Y, Li Y, Wang Ga and Peng M (2016) Genome-wide identification of cassava R2R3 MYB family genes related to abscission zone separation after environmental-stress-induced abscission. Sci Rep 6:32006.

Libault M, Joshi T, Takahashi K, Hurley-Sommer A,Puricelli K,Blake S,Finger RE,Taylor CG,Xu D,Nguyen HT et al. (2009) Large-scale analysis of putative soybean regulatory gene expression identifies a Myb gene involved in soybean nodule development. Plant Physiol 151:1207-1220.

Lippold F, Sanchez DH, Musialak M, Schlereth A, Scheible WR, Hincha DK and Udvardi MK (2009) AtMyb41 regulates transcriptional and metabolic responses to osmotic stress in Arabidopsis. Plant Physiol 149:1761-1772.

Lipsick JS (1996) One billion years of Myb. Oncogene 13:223-235.

Martin C and Pazares J (1997) MYB transcription factors in plants. Trends Genet 13:67-73.

Ogata K, Morikawa S, Nakamura H, Sekikawa A, Inoue T, Kanai H, Sarai A, Ischii S and Nishimura Y (1994) Solution structure of a specific DNA complex of the Myb DNA-binding domain with cooperative recognition helices. Cell 79:639-648.

Ogata K, Morikawa S, Nakamura H, Hojo H, Yoshimura S,Zhang R,Aimoto S,Ametani Y,Hirata Z,Sarai A et al. (1995) Comparison of the free and DNA-complexed forms of the DNA-binding domain from c-Myb. Nat Struct Biol 2:309-320.

Poole RL (2007) The TAIR database. Methods Mol Biol 406:179-212.

Prabu G and Prasad DT (2012) Functional characterization of sugarcane MYB transcription factor gene promoter (PScMYBAS1) in response to abiotic stresses and hormones. Plant Cell Rep 31:661-669.

Romero I, Fuertes A, Benito MJ, Malpica JM, Leyva A and Paz-Ares J (1998) More than 80 regulatory genes in the genome of Arabidopsis thaliana. Plant J 14:273-284.

Rosinski JA and Atchley WR (1998) Molecular evolution of the Myb family of transcription factors: evidence for polyphyletic origin. J Mol Evol 46:74-83.

Seo PJ, Xiang F, Qiao M, Parket JY, Lee YN, Kim SG, Lee YH, Park WJ and Park CM (2009) The MYB96 transcription fac- tor mediates abscisic acid signaling during drought stress response in Arabidopsis. Plant Physiol 151:275-289.

Shu Y, Liu Y, Zhang J, Song L and Guo C (2016) Genome-Wide analysis of the AP2/ERF superfamily genes and their responses to abiotic stress in Medicago truncatula. Front Plant Sci 6:1247.

Stracke R, Werber M and Weisshaar B (2001) The R2R3-MYB gene family in Arabidopsis thaliana. Curr Opin Plant Biol $4: 447-456$.

Tamura K, Peterson D, Peterson N, Stecher G, Nei M and Kumar S (2011) MEGA5: Molecular Evolutionary Genetics Analysis using maximum likelihood, evolutionary distance, and maximum parsimony methods. Mol Biol Evol 28:2731-2739.

Trapnell C, Pachter L and Salzberg SL (2009) TopHat: Discovering splice junctions with RNA-Seq. Bioinformatics 25:1105-1111.

Trapnell C, Roberts A, Goff L, Pertea G, Kim D, Kelley DR, Pimentel H, Salzberg SL, Rinn JL and Pachter L (2012) Differential gene and transcript expression analysis of RNA-seq experiments with TopHat and Cufflinks. Nat Protoc 7:562-578.

Urao T, Shinozaki K, Urao S and Shinozaki K (1993) An Arabidopsis myb homolog is induced by dehydration stress and its gene product binds to the conserved MYB recognition sequence. Plant Cell 5:1529-1539.

Wilkins O, Nahal H, Foong J, Provart NJ and Campbell MM (2009) Expansion and diversification of the Populus R2R3-MYB family of transcription factors. Plant Physiol 149:981-993.

Yanhui C,Xiaoyuan Y,Kun H,Meihua L,Jigang L,Zhaofeng G,Zhiqiang L,Yunfei Z,Xiaoxiao W,Xiaoming Qet al. (2006) The MYB transcription factor superfamily of Arabidopsis: expression analysis and phylogenetic comparison with the rice MYB family. Plant Mol Biol 60:107-124.

Young ND, Debellé F, Oldroyd GE, Geurts R, Cannon SB, Udvardi MK, Benedito VA, Mayer KF, Gouzy J, Schoof H et al. (2011) The Medicago genome provides insight into the evolution of rhizobial symbioses. Nature 480:520-524.

Young ND and Udvardi M (2009) Translating Medicago truncatula genomics to crop legumes. Curr Opin Plant Biol 12:193-201.

Zhang W, Xu F and Cheng S (2017) Characterization and functional analysis of a MYB gene (GbMYBFL) related to flavonoid accumulation in Ginkgo biloba. Genes Genomics 49:49-61.

Zhao P, Li Q, Li J, Wang L and Ren Z (2014) Genome-wide identification and characterization of R2R3MYB family in Solanum lycopersicum. Mol Genet Genomics 289:1183-1207.

Zhu N, Cheng S, Liu X, Du H, Dai M, Zhou DX, Yang W and Zhao Y (2015) The R2R3-type MYB gene OsMYB91 has a function in coordinating plant growth and salt stress tolerance in rice. Plant Sci 236:146-156.

\section{Supplementary material}

The following material is available for this article:

Table S1 - PCR primers used for quantitative real-time PCR analysis. 
Table S2 - Expression data of R2R3-MYB transcription factors in various tissues and development processes.

Table S3 - Expression data of R2R3-MYB transcription factors in various tissues.
Table S4 - Expression data of R2R3-MYB transcription factors response to abiotic stresses.

Associate Editor: Hong Luo

License information: This is an open-access article distributed under the terms of the Creative Commons Attribution License (type CC-BY), which permits unrestricted use, distribution and reproduction in any medium, provided the original article is properly cited. 